\title{
REMARKS ON MINIMAL ROUND FUNCTIONS
}

\author{
GEORGI KHIMSHIASHVILI \\ Georgian Academy of Sciences \\ A. Razmadze Mathematical Institute \\ Aleksidze str. 1, 380093 Tbilisi, Georgia \\ E-mail:khimsh@rmi.acnet.ge \\ DIRK SIERSMA \\ Department of Mathematics, Utrecht University \\ P.O.Box 80010, 3508 TA Utrecht, The Netherlands \\ E-mail: D.Siersma@math.uu.nl
}

\begin{abstract}
We describe the structure of minimal round functions on compact closed surfaces and three-dimensional manifolds. The minimal possible number of critical loops is determined and typical non-equisingular round function germs are interpreted in the spirit of isolated line singularities. We also discuss a version of Lusternik-Schnirelmann theory suitable for round functions.
\end{abstract}

1. Introduction. A differentiable function on a differentiable manifold is called a round function if its critical set is a union of embedded one-dimensional submanifolds. In general it is not assumed that the critical submanifolds are non-degenerate critical submanifolds in the sense of R. Bott [3] so this is a straightforward extension of the notion of round Morse function introduced by W. Thurston [30].

Our main concern in this paper are round functions on a given compact closed (i.e., without boundary) smooth manifold with the minimal possible number of critical submanifolds (critical loops). By analogy with the terminology of [29] they will be called minimal round functions as in [21]. We are especially interested in describing possible changes of the topology of their Lebesgue sets (preimages of semi-infinite intervals) and typical local models of their singular behaviour near critical loops. Our setting and approach are much in the spirit of F. Takens' paper [29] which contains a comprehensive

2000 Mathematics Subject Classification: Primary 57R70; secondary 55M30.

Key words and phrases: Round function, round Morse function, equisingular critical loop, isolated line singularity, Lusternik-Schnirelmann category.

The paper is in final form and no version of it will be published elsewhere. 
treatment of similar questions for functions with isolated critical points.

It should be noted that unlike to the round Morse functions which gained a lot of attention [1], [30], [24], [8], round functions with degenerate critical loops (called below degenerate round functions) are rather poorly understood. For example, it is still unclear how to describe the class of compact closed manifolds which possess round functions. Some results about general (not necessarily Morse) round functions may be found in [1], $[2],[24],[26]$, but to the best of our knowledge there exists no systematic exposition of this topic in the literature. The present paper may be considered as an attempt to fill this gap and provide a framework for further investigations.

As we were able to conclude from [2], [24], [26] and discussions with colleagues, many natural questions about round functions remain unanswered even in low dimensions. Thus it seemed reasonable to begin by discussing round functions on low-dimensional manifolds. Specifically, we consider round functions on surfaces (two-dimensional smooth manifolds) and 3-manifolds (three-dimensional smooth manifolds) and their local behaviour near critical submanifolds. Some of these results are rather simple and we do not exclude that they may be known for the experts or even belong to "mathematical folklore", but we have good evidence to believe that in any case our presentation contains certain novelties arising from the treatment of round functions from the singularity theory viewpoint in the spirit of isolated line singularities [27], [28].

One of the basic ideas we want to formulate and illustrate here, is that round functions with degenerate critical loops appear quite naturally in a certain simple context and their transversal singular behaviour along critical loops resembles some patterns exhibited by the so-called isolated line singularities [27]. The context we have in mind is related to certain homotopy invariants similar to the classical Lusternik-Schnirelmann category [18] and invariants considered by F. Takens [29]. We describe this setting in some detail as well as typical examples of degenerate round functions in low dimensions.

In order to endow the whole topic with a proper background, we first address the general existence problem for round functions on closed manifolds. We recall main results in this direction from [1], [23] and complement them by some observations about the Euler characteristic of Lebesgue sets. Results of the first section imply, in particular, that degenerate round functions, generally speaking, cannot be approximated by round Morse functions. This shows that degenerate round functions are in some sense inevitable and should be studied by themselves.

We proceed by considering examples of minimal round functions on compact closed surfaces. It turns out that, in the orientable case, critical loops of such functions are transversally non-degenerate except at a finite number of points of Whitney umbrella type $\left(D_{\infty}\right.$-points in notation of [27], [28]). For a round function with critical loops of such type, we describe possible changes in topology of Lebesgue sets under passing of a critical level. This enables us, in particular, to determine the minimal possible number of $D_{\infty}$-points on a given orientable surface. We also find out that round functions exist on any closed 3-manifold, which is in a contrast with the fact that not all of 3-manifolds possess round Morse functions [24].

Thus we achieve a rather detailed description of minimal round functions on closed 
surfaces. These two-dimensional results serve as one of the main paradigms for our research and suggest some approaches to higher-dimensional cases although it should be noted that in higher dimensions the situation is much more complicated. In particular, it becomes hard to compute the relevant invariants, like the round complexity, by visual geometric considerations so one has to develop some topological machinery suitable for this purpose.

With this in mind, we describe some tools sufficient to obtain lower estimates for the number of critical loops. As is well known, the classical Lusternik-Schnirelmann category gives a lower estimate for the minimal number of isolated critical points of a smooth function on a given manifold [18]. We follow the same pattern in the context of round functions by using an appropriate version of generalized category-like invariants introduced by M. Clapp and D. Puppe [4] (cf. also [2], [20]). In such a way we come to lower estimates for the minimal possible number of critical loops in terms of these invariants and conclude the section by computing them in some simple cases.

In the last section we discuss minimal round functions on 3-manifolds. In particular, we present a complete list of (homeomorphy types of) 3-manifolds possessing round functions with two or three critical loops and obtain some corollaries concerned with the computation of round category and round complexity. These results rely on some elaborate results of three-dimensional topology and may be considered as our main new contribution to the topic. A short announcement of some of our results appeared in [21]. We are grateful to J. Gómez-Larrañaga for useful comments and suggestions concerning an earlier version of the manuscript.

The authors acknowledge partial financial support by INTAS, projects 96-713 and 00-259, during the period of joint work on this paper.

2. Definitions and setting. For brevity, throughout the whole text the word "smooth" means "infinitely differentiable". Manifolds are always assumed to be smooth compact and closed (without boundary), those with boundary will be referred to as $\partial$-manifolds.

Let $f: M \rightarrow \mathbf{R}^{1}$ be a smooth function on a (smooth compact) manifold $M$. As usual, the critical set $C(f)$ of function $f$ is defined as the set of all points where differential $d_{p} f$ vanishes. In our situation $C(f)$ is evidently non-empty and carries a lot of topological information about manifold $M$. The latter circumstance is well known and has spectacular manifestations in the case of functions with isolated critical points, especially Morse functions [22]. It should be noted that often it is also necessary (or useful) to consider functions with non-isolated critical points [3], [30], [8].

In many problems of differential topology and Hamiltonian mechanics an important role is played by so-called round Morse functions, with critical sets consisting of several smooth loops which are non-degenerate in the sense of Bott [1], [30]. In some situations the condition of non-degeneracy does not seem natural, so we introduce a more general class of functions. As usual, the term "loop" refers to a continuous image of circle $S^{1}$ and a loop is called simple if it has no self-intersections. In line with that, the image of a smooth embedding of $S^{1}$ into a given manifold $M$ will be called a smooth simple loop in $M$. 
DeFinition 1. Function $f$ is called a round function if its critical set $C(f)$ is a disjoint union of several smooth simple loops. Components of $C(f)$ are called critical loops of $f$. If all critical loops of $f$ are non-degenerate in the sense of Bott, then $f$ is called a round Morse function [30]. If all critical loops are non-degenerate except a finite number of points on them, we will say that $f$ is an almost Morse round function.

Examples of round functions are immediate, but unlike to Morse functions, they do not exist on all manifolds.

Proposition 1. No round functions exist on two-sphere $S^{2}$ and real projective plane $\mathbf{R P}^{2}$.

Indeed, on $S^{2}$ any critical loop bounds an embedded disc in interior of which the function in question evidently should have further critical points (maxima or minima, at least). On the other hand, by an evident compactness argument one always finds a critical loop containing no other critical loops in its interior. So there should exist some isolated minima or maxima in its interior, which contradicts the definition of round function. In the case of $\mathbf{R P}^{2}$ one arrives at the desired conclusion by considering lifts of functions to the universal covering space $S^{2}$.

At the same time there are many evident examples of round functions in all dimensions. For example, on any manifold of the form $M \times S^{1}$ round functions arise from arbitrary functions with isolated critical points on $M$. The same holds for circle bundles over manifolds, which provides, in particular, round functions on odd-dimensional spheres from Hopf fibrations $S^{2 n+1} \rightarrow \mathbf{C P}^{n}$, the most visual example being a well-known function ("height-Hopf") on $S^{3}$ having two critical loops which are linked (as fibres of the Hopf fibration $S^{3} \rightarrow S^{2}$ ).

Thus the existence issue for round functions is not completely trivial and we will present some more comments upon it in the sequel. Note that in three-dimensional case the mutual position of critical loops is also a non-trivial issue.

Proposition 2. On $S^{3}$ there are no functions with two critical loops which are unlinked.

Indeed, any function with two critical loops gives a decomposition of $S^{3}$ in a union of two solid tori $S^{1} \times D^{2}$ (which are suitable tubular neighbourhoods of critical loops) glued by certain diffeomorphism of their boundaries. Clearly, only homotopy classes of gluing diffeomorphisms are important and those are known to be described by elements of $S L(2, \mathbf{Z})$ expressing the effect of gluing on $H_{1}\left(T^{2}, \mathbf{Z}\right)$ [17]. Discussion in [17] shows that the result of such gluing is diffeomorphic to $S^{3}$ if and only if the core circles of those solid tori are linked with linking number \pm 1 (in other cases one obtains $S^{1} \times S^{2}$ or such lens spaces which are not even homeomorphic to $S^{3}$ ).

On the other hand, it is clear that round functions with unlinked critical circles exist on direct products of the form $M^{2} \times S^{1}$. These simple observations already suggest some natural problems which seem to be open.

Problem 1. Characterize closed 3-manifolds which possess round functions with unlinked critical circles. 
Problem 2. For a given compact connected 3-manifold, characterize links which may be represented as critical sets of round functions.

We would like to point out that results of [15] provide many examples of links representable as critical sets of round functions on $S^{3}$. Actually, we believe that the second problem in the case of three-sphere can be completely investigated by methods of [15]. Using Bott's theory of non-degenerate critical manifolds it is quite simple to indicate a topological invariant responsible for the existence of round Morse functions. Indeed, it is clear that if a non-degenerate critical manifold is homeomorphic to the circle, then under passage of this critical level the Euler characteristic of Lebesgue sets $\{f \leq a\}$ remains unchanged [3]. Thus round Morse functions may only exist on manifolds with vanishing Euler characteristic.

D. Asimov proved that the converse is also true in all dimensions bigger than three [1] (this is not the case in three dimensions because there exist compact closed 3-manifolds which do not possess round Morse functions [24]). As follows from [19], one can generalize these observations by looking at possible changes of the Euler characteristic in the socalled transversally equisingular case. It turns out that the transversally equisingular behaviour is often exhibited by minimal round functions, so we give a precise definition.

DeFinition 2. A function $f$ is called transversally equisingular at a critical submanifold $K$ if, for every point $p \in K$, germs at $p$ of restrictions of $f$ to small discs transversal to $K$ at $p$ belong to the same right-left equivalence class [9].

Obviously, natural examples of equisingular critical submanifolds emerge from fibre bundles. For example, given a smooth circle bundle $\pi: E \rightarrow M$ one gets equisingular round functions on $E$ by taking compositions $f \cdot \pi$, where $f$ is any function with isolated critical points on $M$. In particular, if $f$ is a Morse function then $f \cdot \pi$ is a round Morse function.

Proposition 3 ([19]). If $K$ is a transversally equisingular critical submanifold of function $f$ then, under the passage of level $f(K)$, the Euler characteristic of Lebesgue set $\chi(\{f \leq a\})$ is changed by an integer multiple of $\chi(K)$.

Corollary 1. Equisingular round functions exist on a manifold $M$ if and only if $\chi(M)=0$.

In order to prove this proposition, one uses the multiplicative property of the Euler characteristic and existence of a locally trivial fibration structure in a neighbourhood of an equisingular critical submanifold (compare similar statements in [6]), to show that the total change of the Euler characteristic of Lebesgue set $\triangle \chi\{f \leq a\}$ is equal to the product $\triangle \chi($ slice $) \cdot \triangle \chi(K)$. Actually, it is not difficult to show that in this case $\triangle \chi$ (slice) is equal to the $\operatorname{gradient}$ index $\operatorname{ind}_{p} \operatorname{grad}\left(f \mid D_{p}\right)$ of restrictions of $f$ to small transversal $\operatorname{discs} D_{p}$ at $p$.

For further reference, it is also convenient to introduce another natural class of degenerate round functions.

Definition 3. A point $p \in K$ on a critical loop $K$ of a round function $f$ is called a point of $D_{\infty}$-type (for $f$ ) if, in some system of local coordinates $\left(x_{1}, \ldots, x_{n}\right)$ around $p$, 
function $f$ takes the form $x_{1} x_{2}^{2} \pm x_{3}^{2} \pm \ldots \pm x_{n}^{2}$. A critical loop $K$ is called a Morse-Whitney critical loop if $f$ is transversally non-degenerate on $K$ with only possible exception of a finite number of points which are all of $D_{\infty}$-type. A round function is called a MorseWhitney function if all of its critical loops are Morse-Whitney critical loops.

In this paper we are basically concerned with estimating the minimal possible number of critical loops of a round function on a given manifold. Recall that F. Takens in [29] introduced an interesting topological invariant $F .(M)$ of a smooth manifold $M$, defined as the minimal number of critical points of smooth functions on $M$. In some cases he was able to show that $F .(M)$ coincided with the Lusternik-Schnirelmann category cat $M$, and explicitly constructed so-called exact functions which have precisely cat $M$ critical points on $M$. Our strategy is to mimic his approach in the context of round functions.

Definition 4. Round complexity $\operatorname{roc} M$ of a manifold $M$ is defined as the minimal possible number of critical loops of round functions on $M$. If round functions on $M$ do not exist, we put $\operatorname{roc} M=\infty$. A round function $f$ is called a minimal round function if the number of components of $C(f)$ is equal to $\operatorname{roc} M$.

Round complexities are usually hard to compute. Below we will introduce an appropriate homotopy invariant of $M$, round category Tcat $M$, which gives a lower estimate for $\operatorname{roc} M$. We will be interested in finding situations in which $\operatorname{roc} M=$ Tcat $M$. If this is the case, we will say that a round function is exact if it has precisely Tcat $M$ critical loops. In the sequel we are mainly concerned with minimal and exact round functions on surfaces and 3-manifolds.

3. Round functions in low dimensions. We describe now some constructions of round functions on surfaces and 3-manifolds and discuss the structure of minimal round functions. Existence of round functions on surfaces and three-manifolds was also stated in the note [2] but neither [2] nor other publications of the same author contain any comments about construction and local behaviour of minimal round functions. A weaker version of the following result was presented in [21].

TheOREM 1 (cf. [2], [21]). Round functions exist on all closed surfaces, except $S^{2}$ and $\mathbf{R P}^{2}$. Transversally equisingular round functions, as well as round Morse functions, exist only on $T^{2}$ and Klein bottle $K^{2}$. The round complexity is equal to two for $T^{2}$ and $K^{2}$, and it is equal to three in all remaining cases. On all closed surfaces there exist almost Morse round functions. On surfaces with the even Euler characteristic there always exist minimal round functions which are Morse-Whitney functions. The minimal number of $D_{\infty}$-points of a minimal Morse-Whitney function on an orientable surface of genus $g$ is equal to $2 g-2$.

We prove this using a sort of surgery suitable for round functions on surfaces the best description of which could be probably given just by drawing some pictures. We prefer nevertheless to present a few concise comments which should make clear the main point of construction. For simplicity, we only consider the orientable case and start with a standard model of a (usual round) torus $T^{2}$ with the evident minimal round function on it ("height function on a lying tyre"). 
We take two such tori and arrange that the circle of maxima on the first copy lies on the same level with the circle of minima on the second copy, say on the zero-level. Then we take a point $P$ on one circle and a point $Q$ on the other, delete small discs around these points and perform our surgery. To this end we glue a cylinder to boundaries of deleted discs and try to extend our function to that cylinder.

A simple visual examination of arising picture shows that this is really possible and the simplest way of doing so is to join "free ends" of original critical circles by two segments on the cylinder with one Whitney umbrella $\left(D_{\infty}\right.$-point) on each of those segments. As one immediately sees, the result is a round function with three critical loops on a connected sum of two tori and it becomes clear that this procedure may be iterated, which yields round functions on all orientable closed surfaces.

A slight modification of the same surgery enables one to fuse any pair of critical loops containing points of different types (max vs. min). This shows that the number of critical loops may be reduced to three, for any orientable surface with the genus higher than one, and the arising round functions are Morse-Whitney functions. Using (an adapted version of) standard Morse theory we can easily check that the number of Whitney umbrellas on every critical loop is even and the Euler characteristic of Lebesgue sets changes by $-2 k$ under passage of a Morse-Whitney loop with $2 k$ points of $D_{\infty}$-type on it, which gives the last statement of the theorem.

In the non-orientable case one can develop similar surgery which uses gluing of Möbius bands. It should be noted that critical loops provided by this procedure are of the transversal type $A_{2}$ [27] so in this way one cannot derive existence of almost Morse functions on non-orientable closed surfaces. To prove the latter statement one may apply another natural procedure which uses blow-ups of isolated extrema and produces almost Morse critical loops with points of $J_{2, \infty}$-type, in the notation of [27]. Thus we obtain all statements presented in Theorem 1.

We consider now round functions on 3-manifolds. Here situation is substantially more complicated and our results are less complete. For the sake of clarity we first formulate the existence result. Recall that there exists an especially well understood class of 3-manifolds, the so-called Waldhausen class, which consists of unions of Seifert fibrations patched along parts of their boundaries [17].

Theorem 2 (cf. [2], [21]). Round functions exist on all closed 3-manifolds. Round complexity of a closed 3-manifold does not exceed four. Transversally equisingular round functions, as well as round Morse functions, exist only on 3-manifolds of Waldhausen class.

The simplest way to prove the first statement is to refer to the well known result [17] which states that any 3-manifold $M^{3}$ can be obtained from three-sphere $S^{3}$ by surgery along a certain link $L \subset S^{3}$. This basically amounts to cutting out several solid tori $\mathbf{T}_{i}$ (tubular neighbourhoods of the components of $L$ ) from $S^{3}$ and then gluing them back to the remaining part $Y=\operatorname{cl}\left(S^{3}-\bigcup \mathbf{T}_{i}\right)$ with certain twists defined by diffeomorphisms of their boundaries $T_{i}^{2}$. Granted this, we may start by taking a standard round function $F$ on $S^{3}$ ("height-Hopf") and then properly modify it on the interiors of deleted solid tori to 
obtain a round function on $M^{3}$ with critical loops coinciding with the core circles of solid tori $\mathbf{T}_{i}$. To this end it is sufficient to be able to extend a given function with non-vanishing gradient on the boundary of a solid torus $\mathbf{T}$ to the whole of $\mathbf{T}$ so that the critical set of extension coincides with the core circle. Existence of such an extension on each meridian disk follows from the key technical result of [29] (Theorem 2.7) which we call "Takens trick" for short. The fact that those extensions can be done in such way that one obtains a smooth function on the whole solid torus, is merely an evident "parametrized version of Takens trick" (cf. [6]). In fact, in the case of solid torus existence of the desired extension can also be proven by an elementary direct argument in the spirit of [6].

Furthermore, we notice that another possible way of dealing with 3-manifolds is based on the fact that each 3-manifold is representable as the union of several solid tori glued along parts of their boundaries [17]. Actually, the above reasoning can be performed in terms of unions of solid tori (cf. [16], [10]). Moreover, according to [12], any 3-manifold $M^{3}$ can be obtained by gluing together not more than four solid tori. Since the critical loops of the function constructed by such an extension procedure coincide with the core circles of those solid tori, we conclude that one can always construct a round function with no more than four critical loops, hence the round complexity of $M^{3}$ does not in fact exceed four.

The statement about transversally equisingular round functions is reduced to the particular case of round Morse functions by a procedure of "round morsification" which amounts to saying that each equisingular round function can be approximated by round Morse functions [24]. The statement about round Morse functions follows from the results of [24]. Indeed, in [24] it was shown that a 3-manifold belongs to Waldhausen class if and only if it admits so-called round handle decompositions introduced in [1]. The desired conclusion follows by noticing that, as was established in [23], existence of a round handle decomposition is equivalent to existence of a round Morse function.

REMARK 1. Other instances of application of a "parametrized version of Takens trick" may be found in [6]. We emphasize that results of [6] cannot be automatically applied in our case because they were obtained with the assumption that critical submanifolds are simply connected.

Two important general conclusions which one may derive from the above results are that, first, degenerate round functions are inevitable outside Waldhausen class, and, second, an arbitrary round function not always can be approximated by round Morse functions. In our opinion this provides additional motivation for not restricting one's considerations to round Morse functions. The papers [12], [13], [14] which contain developments in this spirit and refer to some results outlined in our short note [21], also witness in favour of usefulness of the above setting.

4. Round category and cup-length. In higher dimensions, there is already little hope to succeed in studying round functions by purely geometric means so one has to develop some suitable topological tools. We introduce below two relevant notions, round category and round cup-length, and present analogues of the two basic inequalities of Lusternik-Schnirelmann theory (Propositions 4 and 5). Similar notions and results can 
be found in [2] and [20] but actually they are just versions of a general discussion in [4]. We compute these invariants in some cases of interest, including closed surfaces and certain 3-manifolds. Combined with results of Section 3, this proves existence of exact round functions on closed surfaces and certain connected sums of Seifert fibrations.

Recall that a subset $A \subset X$ of a topological space $X$ is called $T$-categorical if the inclusion map $i: A \rightarrow X$ may be factored through the circle $T$ up to homotopy [4], that is, there exist continuous maps $\phi: A \rightarrow T$ and $\psi: T \rightarrow X$ such that $\psi \phi$ is homotopic to $i$.

Definition 5 (cf. [4], [2], [20]). Round category Tcat $X$ of a connected paracompact space $X$ is defined as the minimal possible cardinality of coverings of $X$ by $T$-categorical closed subsets. A round function on manifold $M$ is called an exact round function if the number of its critical loops is equal to Tcat $M$.

This is just a special case of the general definition from [4] so we may use results from [4]. In particular, from the discussion in [4] it follows that, for any closed manifold $M$ which is not homeomorphic to $S^{1}$, one has inequalities: $2 \leq$ Tcat $M \leq$ cat $M \leq \operatorname{dim} M+1$. In particular, it follows that, for any $n \geq 2$, Tcat $S^{n}=2$.

Proposition 4. For a closed manifold $M$, one has Tcat $M \leq \operatorname{roc} M$.

This follows from a more general statement found in [4] (Theorem 2.3) so we omit the proof. It is now easy to verify that Theorem 1 enables one to compute round category for closed surfaces and yields examples of exact round functions.

Corollary 2. Exact round functions exist on all surfaces except two-torus and Klein bottle.

For evident reasons, it is tempting to compare round category of a circle bundle with the usual Lusternik-Schnirelmann category of its base. It may be proven that for a circle bundle $E$ over a closed surface $M$, one always has Tcat $E=$ cat $M$ (this is actually a direct consequence of our Theorem 3 in the next section, as the reader can easily verify). There is little hope that the same holds if the base is an arbitrary CW-complex but there is good evidence that this is true for direct products with the circle.

Problem 3. Prove that $\operatorname{Tcat}\left(X \times S^{1}\right)=$ cat $X$ if $X$ is an arbitrary two-dimensional CW-complex.

It seems that in higher dimensions this equality cannot hold for all smooth manifolds, so looking for a corresponding counter-example might be a reasonable enterprise. In general, computation of round category in higher-dimensional cases cannot usually be done by purely geometric considerations like the ones used in the proofs of Theorems 1 and 2. Some tools from algebraic topology are helpful here and we borrow one of them from $[4]$.

Definition 6 ([4]). Round cup-length $\operatorname{Tcl}(X)$ of a topological space $X$ is defined as the nilpotency index [18] of the subring $H_{T}^{*}(X, \mathbf{Z})$ equal to the intersection of all kernels of induced mappings $F^{*}: H^{*}(X, \mathbf{Z}) \rightarrow \mathbf{Z}$, where $F: T \rightarrow X$ runs over all continuous mappings of the circle $T$ into $X$. 
Proposition 5. For any $C W$-complex $X$, one has $\operatorname{Tcl}(X)+1 \leq \operatorname{Tcat} X$.

As in the case of Proposition 4, the proof is obtained by a simple modification of the proof of a similar statement in [4] (Proposition 3.1) and is therefore omitted. This result enables one to compute the round category in some higher-dimensional cases. We only present two results of this kind which illustrate some general phenomena exhibited by these invariants.

Proposition 6. $\operatorname{roc} S^{5}=3$.

Since it is easy to construct a round function with three critical loops on $S^{5}$ from fibration $S^{5} \rightarrow \mathbf{C P}^{2}$, it is sufficient to show that $S^{5}$ admits no round functions with just two critical loops. The latter fact can be proven by analyzing possible topological types of unions of two copies of $S^{1} \times D^{4}$ glued along some diffeomorphism of their boundaries. Namely, it is not difficult to show using van Kampen's theorem that the result of such gluing can never have the homotopy type of $S^{5}$.

Proposition 7. $\operatorname{roc} T^{n}=$ Tcat $T^{n}=n$.

This follows by first showing that Tcat $T^{n} \geq n$ and then constructing a round function with $n$ critical loops. An elementary examination of cup-products in $H^{*}\left(T^{n}\right)$ shows that $\operatorname{Tcl}\left(T^{n}\right)=n-1$ (cf. [4]), and it is also easy to obtain a desired function on $T^{n}$. Indeed, it is well known that cat $T^{n-1}=n[18]$ and one easily shows by induction that a function with exactly $n$ isolated critical points exists on $T^{n-1}$.

Thus exact round functions exist on all tori, while $S^{5}$ admits no exact round functions. Hence the inequality in Proposition 4 cannot be substituted by equality and of course the same refers to the inequality in Proposition 5. Nevertheless, we know many cases when it is possible to prove that one or both of these equalities take place, so this issue deserves further investigation.

REMARK 2. It is also easy to prove that, for all $n \geq 3$, roc $S^{2 n-1} \geq 3$ by a similar topological analysis of unions of two copies of $S^{1} \times D^{2 n-2}$. Actually, one can even compute the round complexity in question and show that, for all $n \geq 2, \operatorname{roc} S^{2 n-1}=n$. We do not make here any attempts to describe the proof, since the argument which we possess, relies on results of Conley index theory which do not seem appropriate to be discussed here.

5. Minimal round functions on 3-manifolds. In order to formulate the last main result, we need some notation and conventions. For brevity, we only discuss orientable 3-manifolds.

The symbol \# will always denote the connected sum of two closed 3 -manifolds. By $B_{j}$ we denote a copy of the product $S^{1} \times S^{2}$ and by $B=\# B_{j}$ an arbitrary finite connected sum of such products. By $L_{j}$ or simply $L$ we denote any lens space with a non-trivial finite fundamental group. Finally, let Sf(3) denote any Seifert fibration [17] with no more than three exceptional fibres.

TheOREM 3. Let $M$ be an orientable compact closed 3-manifold. If roc $M$ is equal to two, then $M$ is homeomorphic to a lens space. If $\operatorname{roc} M$ is equal to three, then $M$ is 
homeomorphic to one of the manifolds of the following type:

$$
L \# B, L_{1} \# L_{2}, L_{1} \# L_{2} \# B, L_{1} \# L_{2} \# L_{3}, L_{1} \# L_{2} \# L_{3} \# B, \operatorname{Sf}(3), \operatorname{Sf}(3) \# B .
$$

From this result it follows, in particular, that the upper bound four for round category of 3-manifolds established in Theorem 2, is exact.

Corollary 3. There exist 3-manifolds $M$ with $\operatorname{roc} M=4$ and such examples can be found within the Waldhausen class.

REMARK 3. Theorem 3 enables one to compute the round complexity for many 3-manifolds and may be considered as an analogue of Theorem 3.3 from [29]. Despite apparent similarity of formulations of these two results, their proofs use essentially different techniques. In particular, we make no use of so-called fillings [29] playing the crucial role in Takens' approach.

The proof of Theorem 3 makes an essential use of the existing comprehensive structural theory of 3-manifolds [17]. The crucial ingredient is an analysis of possible homeomorphy types of unions of several solid tori in the spirit of [16]. First, applying an appropriate modification of Morse theory we show that a manifold with the round complexity not exceeding three is representable as a union of two or three solid tori appearing as suitable tubular neighbourhoods of critical loops. Results of [16] actually contain the topological classification for certain types of unions of two solid tori, and with some additional effort we are able to show that they are applicable in our situation. It remains to extend the classification to unions of three solid tori, which is done in an analogous way by making proper use of results of [10].

REMARK 4. A straightforward attempt to prove that in situation of Theorem 3, a manifold $M$ is actually diffeomorphic to one of the manifolds in these lists, meets some serious difficulties typical for low-dimensional differential topology. The situation here is analogous with [29] since availability of classification of 3-manifolds $M$ with $F .(M)=2$ depends on validity of the Poincaré conjecture. If, as the recent results of G. Perelman [25] enable one to hope, the Poincaré conjecture appears true, this will also simplify some of the issues related to round category and make our "caveat" unnecessary.

Thus it turns out that the round category is more hard to compute than the round complexity. Nevertheless, Theorem 3 apparently computes the round category of any 3-manifold $M$ with $\operatorname{roc} M \leq 3$. It is also possible to develop explicit constructions of minimal round functions on many 3-manifolds from the above lists and obtain some information on the transversal behaviour of resulting functions along their critical loops.

Note that one should indeed check existence of the desired round functions on 3-manifolds from the above lists, since the latters are only homeomorphic to 3-manifolds on which the existence of a minimal round function is granted, and we do not have a proof of the fact that $\operatorname{roc} M$ is a topological invariant. We construct desired round functions by using an extension of surgery applied in the proof of Theorem 1. Such surgery enables one, in particular, to fuse two extremal loops of different types (a "max-min pair") lying on the same critical level in such a way that the result is again a round function with only two exceptional points on the corresponding critical loop. This leads to an induc- 
tive construction of round functions on 3-manifolds from our lists and yields exact round functions on some of them but the results are not yet complete.

Proposition 8. All 3-manifolds from the above lists possess almost Morse round functions.

One can prove this by checking that our surgery produces an almost Morse round function on a connected sum of two 3-manifolds from a pair of almost Morse round functions on summands.

REMARK 5. We want to emphasize that existence of exact round functions on the above 3-manifolds cannot be discussed since we have not yet computed their round categories. Moreover, even their round complexities should be computed by a separate argument since we do not know if round complexity is a topological invariant (from the definition it only follows that round complexity is an invariant of diffeomorphic type).

Thus it remains unclear if exact round functions exist on all 3-manifolds. The latter fact would be established, if the following problem has positive solution.

Problem 4. Prove that for a compact closed 3-manifold $M$, Tcat $M=3$ if and only if $\operatorname{roc} M=3$.

As a natural first step, one should of course try to compute round categories for 3 -manifolds in our lists. It is quite simple to see that the 3-manifolds with round complexity equal to two, have the round category also equal to two. We have checked that the round category is equal to three for some manifolds from our second list, but we do not see any "a priori" reason why the same should hold in remaining cases so the whole issue remains open. In general, round category is difficult to compute and the situation here seems much more complicated than with the classical Lusternik-Schnirelmann category. For example, the Lusternik-Schnirelmann category of a 3-manifold can be read off its fundamental group [11], but we are not aware of any results of such type for the round category.

In order to keep the exposition within a reasonable length we consciously omitted some closely related results and topics. In particular, most of our results have natural generalizations for low-dimensional $\partial$-manifolds and some other types of stratified spaces. Another promising line of development is related with functions on manifolds endowed with codimension-one foliations [7] (cf. also [5]). Moreover, some aspects of the discussion above fit into a more general context of round mappings, i.e., mappings with smooth one-dimensional singular sets. We postpone discussion of those topics for forthcoming publications.

\section{References}

[1] D. Asimov, Round handles and non-singular Morse-Smale flows, Ann. of Math. (2) 104 (1976), 41-54.

[2] O. P. Bondar', On the number of critical submanifolds of a function on a manifold, Ukraïn. Mat. Zh. 45 (1993), 1702-1705 (Russian); English transl.: Ukrainian Math. J. 45 (1993), 1915-1919. 
[3] R. Bott, Nondegenerate critical manifolds, Ann. of Math. (2) 60 (1954), 248-261.

[4] M. Clapp, D. Puppe, Invariants of the Lusternik-Schnirelmann type and the topology of critical sets, Trans. Amer. Math. Soc. 298 (1986), 603-620.

[5] H. Colman, S. Hurder, Tangential LS category and cohomology for foliations, in: LusternikSchnirelmann Category and Related Topics, Contemp. Math. 316, Amer. Math. Soc., Providence, 2002, 41-64.

[6] O. Cornea, Cone-decompositions and degenerate critical points, Proc. Lond. Math. Soc. (3) 77 (1998), 437-461.

[7] S. Ferry, A. Wasserman, Morse theory for codimension-one foliations, Trans. Amer. Math. Soc. 298 (1986), 227-240.

[8] A. T. Fomenko, Morse theory for integrable Hamiltonian systems, Dokl. Akad. Nauk SSSR 287 (1986), 1071-1075 (Russian); English transl.: Soviet Math. Dokl. 33 (1986), 502-506.

[9] M. Golubitsky, V. Guillemin, Stable Mappings and their Singularities, Graduate Texts in Math. 14, Springer, New York, 1973.

[10] J. C. Gómez-Larrañaga, 3-manifolds which are unions of three solid tori, Manuscripta Math. 59 (1987), 325-330.

[11] J. C. Gómez-Larrañaga, F. González-Acuña, Lusternik-Schnirelmann category of 3-manifolds, Topology 31 (1992), 791-800.

[12] J. C. Gómez-Larrañaga, W. Heil, Seifert-Takens-Singhof fillings of 3-manifolds, Topology Proc. 26 (2001/2002), 187-197.

[13] J. C. Gómez-Larrañaga, W. Heil, Seifert unions of solid tori, Math. Z. 240 (2002), 767-785.

[14] J. C. Gómez-Larrañaga, W. Heil, Singhof fillings of closed 3-manifolds, Manuscripta Math. 107 (2002), 15-23.

[15] M. Grayson, C. Pugh, Critical sets in 3-space, Inst. Hautes Études Sci. Publ. Math. 77 (1993), 5-61.

[16] W. Heil, Three-manifolds which are sums of solid tori and Seifert fiber spaces, Proc. Amer. Math. Soc. 37 (1973), 609-614.

[17] J. Hempel, 3-Manifolds, Ann. of Math. Studies 86, Princeton Univ. Press, Princeton, 1976.

[18] I. M. James, On category, in the sense of Lusternik-Schnirelmann, Topology 17 (1978), $331-348$.

[19] G. Khimshiashvili, The Euler characteristic of a manifold and critical points of finite multiplicity of smooth functions, Trudy Tbiliss. Mat. Inst. Razmadze Akad. Nauk Gruzin. SSR 68 (1982), 123-141 (Russian).

[20] G. Khimshiashvili, Remarks on Calkin PI-algebras and category-like invariants, Proc. A. Razmadze Math. Inst. 104 (1994), 95-109.

[21] G. Khimshiashvili, D. Siersma, Minimal round functions on low-dimensional manifolds, Bull. Georgian Acad. Sci. 162:4 (2000), 3-7.

[22] J. Milnor, Morse Theory, Ann. of Math. Studies 51, Princeton Univ. Press, Princeton, 1963.

[23] S. Miyoshi, Foliated round surgery of codimension-one foliated manifolds, Topology 21 (1982), 245-261.

[24] J. W. Morgan, Nonsingular Morse-Smale flows on three-dimensional manifolds, Topology 18 (1979), 41-53.

[25] G. Perelman, Ricci flow with surgery on three-manifolds, arXiv: math.DG/0303109, 22 pp.

[26] V. V. Sharko, Functions on Manifolds. Algebraic and Topological Aspects, Naukova Dumka, Kiev, 1990 (Russian); English transl.: Transl. Math. Monogr. 131, Amer. Math. Soc., Providence, 1993. 
[27] D. Siersma, Isolated line singularities, in: Singularities, Part 2, Proc. Sympos. Pure Math. 40, Amer. Math. Soc., Providence, 1983, 485-496.

[28] D. Siersma, The vanishing topology of non isolated singularities, in: New Developments in Singularity Theory (Cambridge, 2000), Kluwer, Dordrecht, 2001, 447-472.

[29] F. Takens, The minimal number of critical points of a function on a compact manifold and the Lusternik-Schnirelmann category, Invent. Math. 6 (1968), 197-244.

[30] W. Thurston, Existence of codimension-one foliations, Ann. of Math. (2) 104 (1976), 249-268. 